\title{
INFLUÊNCIA DE SISTEMAS DE TERMINAÇÃO DE CORDEIROS SOBRE A PRODUÇÃO E CONDIÇÃO SANITÁRIA DAS OVELHAS EM PASTAGEM
}

\author{
Fernando Hentz¹, Odilei Rogerio Prado¹, Alda Lucia Gomes Monteiro', Damaris \\ Ferreira de Souza ${ }^{1}$, Fernanda Sari Ferreira ${ }^{2}$, Ivan Roque de Barros Filho ${ }^{1}$ \\ 1 UFPR
2 Autônoma
Correspondência: Alda Monteiro: aldaufpr@gmail.com
}

RESUMO: Objetivou-se com esse trabalho avaliar o desempenho, estado corporal, grau de infestação parasitária e a produção e composição do leite de ovelhas com cordeiros em amamentação, em três sistemas de produção de cordeiros para corte. Ovelhas Suffolk e suas respectivas crias foram submetidas aos sistemas de produção: exclusivamente pastagem de azevém; pastagem de azevém com creep feeding ofertado aos cordeiros a $2 \%$ do PC em matéria seca por dia; pastagem de azevém com acesso livre dos cordeiros ao creep grazing com trevo branco. Os piquetes de pastagem eram formados de azevém anual sobressemeado em Tifton-85. Os animais entraram em avaliação aproximadamente 30 dias após o parto. A idade média das ovelhas foi de 4,5 anos, distribuídas de forma homogênea entre os tratamentos. $O$ delineamento experimental foi em blocos ao acaso. Foi observada perda de peso e de condição corporal das ovelhas após o parto, para todos os sistemas de produção, sem diferença $(P>0,05)$ entre os mesmos. O Grau Famacha das ovelhas não variou $(P>0,05)$ entre os tratamentos; entretanto, a infestação parasitária medida pela contagem de ovos por grama de fezes $(\mathrm{OPG})$ foi maior $(P<0,05)$ para as ovelhas com cordeiros que não recebiam suplementação. A produção diária de leite das ovelhas diferiu $(P<0,05)$ entre os tratamentos, sendo menor no sistema em que os cordeiros não foram suplementados. Os teores de gordura, lactose, proteína e sólidos totais do leite não sofreram influência do sistema de produção de cordeiros. A produção de leite se manteve com pouca variação entre a $4^{\underline{a}}$ e $10^{\text {a }}$ semanas pós-parto. A suplementação de cordeiros em creep feeding e creep grazing não influenciou o desempenho ponderal e o estado corporal das ovelhas; porém, o melhor desenvolvimento dos cordeiros suplementados nesses sistemas promoveu a maior produção de leite das mães. O sistema de produção dos cordeiros influenciou o grau de infestação parasitária das matrizes.

Palavras-chave: azevém; cordeiro lactente; creep feeding; creep grazing

\section{LAMB PRODUCTION SYSTEMS AND ITS INFLUENCE ON EWE PRODUCTION AND SANITARY CONDITION IN PASTURE}

\begin{abstract}
Suffolk lactating ewes ( \pm 4.5 years) and their respective lambs were used in a randomized block design with three replications to evaluate if the feeding system of the lambs affects performance, corporal and sanitary condition and ewes milk production and composition. Three different feeding systems were tested: 1) lambs with ewes on ryegrass pasture; 2) lambs with ewes on ryegrass pasture and creep feeding ( $2 \%$ of body weight (BW)/day); and, 3 ) lambs with ewes on ryegrass pasture and creep grazing of white clover. Ryegrass pasture was oversowned on Tifton- 85 . The animals were located in the experimental area 30 days after parturition. Ewes have lost weight and body condition, but there was no significant difference between treatments for that $(P>0.05)$. Ewes sanitary condition evaluated by the FAMACHA method did not differ $(P>0.05)$, but parasitological infection by FEC was higher in those ewes whose lambs were not supplemented. Daily milk production differed between systems and was lower $(P<0.05)$ when lambs were not supplemented. The percentage of fat, lactose, protein and total solids was not affected by systems. Milk production varied slightly and not significantly from $4^{\text {th }}$ to $11^{\text {th }}$ week after parturition. The system of production of lambs affects the productive and sanitary response of lactating ewes.

Supplementation of lambs in creep feeding and creep grazing did not influence weight gain and body condition of ewes, but the better development of lambs supplemented at these systems promoted higher milk production of dams. The production system affected the extent of parasitological infection of the dams.
\end{abstract}

Key Words: creep feeding; creep grazing; lactation; weaning lambs; ryegrass 

das ovelhas em pastagem

\section{INTRODUÇÃO}

Em razão da crescente demanda por carne ovina, principalmente aquela proveniente de animais jovens, tornouse necessária a otimização do crescimento pré-desmama tendo a ovelha papel fundamental na nutrição dos cordeiros desse período (Greenwood et al., 2000). Segundo Poli et al. (2008), o leite materno e/ou presença da mãe foram determinantes no desempenho dos cordeiros em diferentes sistemas de terminação, uma vez que cordeiros desmamados precocemente demoraram a alcançar o peso ideal para abate.

Pensando no custo-benefício da ovinocultura, têm sido avaliados sistemas que promovam a diminuição da idade de abate dos cordeiros visando adicionalmente aumentar a quantidade de partos ao longo da vida produtiva da matriz. Para tal, faz-se necessário 0 cuidado especial com a ovelha na fase de amamentação para assegurar resposta produtiva positiva neste período. A literatura (Poli et al., 2008; Fernandes et al., 2012) tem abordado com frequência o efeito dos sistemas de produção sobre a resposta produtiva dos cordeiros, entretanto pouco se sabe sobre 0 efeito dos sistemas de terminação sobre a reposta produtiva das ovelhas.

A condição corporal da ovelha durante a lactação é influenciada pela oferta de nutrientes (Chiofalo et al., 1993), sendo que a magnitude desta variação está associada em grande parte ao volume de leite produzido. A quantidade e qualidade do leite produzido pela ovelha é afetada, por sua vez, por fatores como o peso da ovelha (Pulina et al., 1994), idade da ovelha (Giaccone et al., 1992), estágio de lactação (Bencini \& Purvis, 1990) e número de fetos nascidos (Bencini et al., 1992; Serra et al., 1993).
Também a contaminação parasitária das matrizes tem relação direta com o seu status nutricional e/ou condição corporal; assim, uma das justificativas para 0 uso de suplementação de crias ao pé da mãe na criação de ruminantes em geral é de que, desta forma, as mães seriam menos procuradas para amamentação, o que levaria à menor secreção de leite e ao menor desgaste físico da matriz. No caso dos ovinos, em especial para as raças nacionais com atividade cíclica ao longo do ano, essa resposta possibilitaria o retorno mais rápido à atividade reprodutiva, resultando em aumento de parições durante a vida útil das ovelhas.

Objetivou-se avaliar a influência de três sistemas de terminação de cordeiros sem desmame, sobre 0 desempenho, infecção parasitária, produção e composição do leite das ovelhas em pastagem de azevém.

\section{MATERIAL E MÉTODOS}

O trabalho foi conduzido no Laboratório de Produção e Pesquisa em Ovinos e Caprinos (LAPOC) na Fazenda Experimental da Universidade Federal do Paraná, localizado na região metropolitana de Curitiba, em PinhaisPR, tendo como coordenadas geográficas 2525' Sul e 498' Oeste, em altitude de $915 \mathrm{~m}$, entre Setembro e Dezembro de 2007. O período experimental teve início 30 dias após o nascimento dos cordeiros e nessa primeira etapa, ovelhas e cordeiros foram mantidos em aprisco ripado, suspenso, sem acesso ao pasto. A diferença máxima de idade entre os cordeiros foi de 7 dias. Vinte e sete ovelhas adultas da raça Suffolk com idade média de 4,5 anos, com parto simples e gemelar foram distribuídas uniformemente em três sistemas: (1) ovelhas e cordeiros lactentes mantidos até 0 abate em pasto de azevém 
(Lolium multiflorum Lam.); (2) ovelhas e cordeiros lactentes mantidos até o abate em pasto de avezém, em creep feeding com ração protéica $(20 \%$ PB e $74 \%$ NDT) à $2 \%$ do peso corporal dos cordeiros em matéria seca por dia; (3) ovelhas e cordeiros lactentes mantidos até 0 abate em pasto de azevém, com acesso livre ao suplemento em creep grazing composto por trevo branco (Tripholium repens).

O experimento foi estabelecido em área de 3,8 ha, dividida em 9 piquetes de 0,4 ha de pastagem de Tifton-85 (Cynodon spp.), sobressemeada com azevém anual (Lolium multiflorum Lam.) em 10 de Abril de 2007, por meio de plantio direto com densidade igual a $60 \mathrm{~kg} / \mathrm{ha}$ de sementes.

A leguminosa trevo branco (Trifolium repens) foi a pastagem suplementar onde somente os cordeiros tinham acesso (creep grazing) sendo estabelecida em área de 0,5 ha. $O$ plantio ocorreu em Março de 2006 com $6 \mathrm{~kg} / \mathrm{ha}$ de sementes. A área foi isolada com tela e dividida em três piquetes de 0,2 ha, referentes às repetições do tratamento em creep grazing.

No período experimental atingiu-se o objetivo de não permitir diferenças quanto à pastagem entre os sistemas, e a caracterização das pastagens é apresentada na Tabela 1.

TABELA 1 - Médias e desvio-padrão para características da pastagem de azevém anual sobressemeada em Tifton- 85 nos três sistemas de producão.

Variável Altura (cm)

LF (kg de MS/ha) $\quad 979,5 \pm 151,1 \quad 795,0 \pm 160,3 \quad 837,8 \pm 142,4$

$\begin{array}{llll}\mathrm{CB}(\mathrm{kg} \text { de M S/ha) } & 1.519,4 \pm 205,2 & 1.223,8 \pm 153,1 & 1.223,6 \pm 139,0\end{array}$

MM (kg de MS/ha) $\quad 1.485,3 \pm 244,7 \quad 1.407,4 \pm 219,3 \quad 1.356,2 \pm 190,2$

Relação folha:colmo $\quad 0,69 \pm 0,08 \quad 0,71 \pm 0,12 \quad 0,75 \pm 0,13$

$\mathrm{MF}=$ mass s de forragem, $\mathrm{LF}=$ lâminas foliares, $\mathrm{CB}=$ colmo + bainha, $\mathrm{MM}=$ mater ial morto.

O delineamento experimental foi em blocos ao acaso com três repetições (piquetes) por tratamento. Cada piquete manteve três ovelhas e quatro cordeiros: duas ovelhas de parto simples - uma ovelha com cordeiro macho e outra com fêmea - e uma ovelha de parto gemelar (um cordeiro macho e uma fêmea). O método de utilização da pastagem foi o de pastejo contínuo com lotação variável (Mott \& Lucas, 1952), sendo que o trevo branco permaneceu restrito ao pastejo dos cordeiros (creep grazing). As unidades experimentais foram constituídas de nove piquetes principais com 0,42 ha de azevém separados por cerca eletrificada. Três destes possuíam entrada para piquete acessório único de trevo branco em 0,17 ha, também com cerca elétrica e tela, onde apenas os cordeiros tinham acesso, por aberturas laterais de $50 \mathrm{~cm}$ de altura por 17 a 22 $\mathrm{cm}$ de largura. Outros três piquetes continham alimentadores privativos, cobertos, construídos em madeira com aberturas laterais de $17 \mathrm{~cm}$ que permitiam somente 0 acesso dos cordeiros ao alimento concentrado fornecido.

Os animais foram distribuídos homogeneamente nos tratamentos em função do peso dos cordeiros. Desta forma, a média de peso no início do experimento foi de $15,52 \mathrm{~kg}$ para os cordeiros sem suplementação, $15,50 \mathrm{~kg}$ para os cordeiros com creep feeding e $15,41 \mathrm{~kg}$ para o creep grazing. As ovelhas entraram no experimento com peso médio inicial de 63,06; 73,17 e $70,38 \mathrm{~kg}$ nos tratamentos sem suplementação, com creep feeding e com creep grazing, respectivamente (Tabela 2). Quanto à infecção parasitária, todos os cordeiros entraram nos piquetes com exame coproparasitológico nulo. Dentre as ovelhas, apenas duas foram everminadas (1400 ovos por grama de fezes - OPG) com princípio ativo Nitroxinil a 34\%, 20 dias antes do início do experimento; no exame de fezes, repetido uma semana antes da entrada na área experimental essas ovelhas apresentaram contagem de OPG igual a zero, como as demais. 

das ovelhas em pastagem

Uma semana antes do início das avaliações, os animais foram encaminhados aos respectivos piquetes para adaptação. Seguindo recomendações de Carvalho et al. (2001) para o melhor desempenho de cordeiros, o azevém foi mantido com altura entre $14-16 \mathrm{~cm}$ (Tabela 1), sendo esta mensurada a cada 14 dias com auxílio de bastão medidor graduado (sward stick) conforme metodologia de Barthram (1986). Os animais testes permaneceram nos piquetes durante todo o período experimental até o abate dos cordeiros machos ou, no caso das fêmeas, até a separação destas para os lotes de reposição (ambos aos $33 \mathrm{~kg}$ de PV).

Semanalmente foram realizadas avaliações do escore de condição corporal (ECC) e pesagem dos animais em balança mecânica, após dieta hídrica de 16 horas. O ECC foi avaliado com os animais em estação, palpandose a rugosidade dos processos transversos e dorsais das vértebras lombares, e atribuindo-se escores de 1 a 5 , sendo 1 representativo de baixo escore e 5 de alto escore corporal, segundo o método proposto por Russel et al. (1969).

\section{Exames coproparasitológicos}

foram efetuados a cada 14 dias seguindo a metodologia modificada de Gordon e Whitlock (1939). As ovelhas e os cordeiros que apresentavam contagem de OPG igual ou superior a 700 foram everminados com o princípio ativo Nitroxinil a $34 \%$, que apresentava eficácia comprovada no rebanho (92\%), na dose de 2,0 mL/50 kg. O método Famacha (Molento et al., 2004) foi aplicado a cada 14 dias apenas para o monitoramento da condição sanitária dos animais, uma vez que a coprocultura realizada no início do experimento revelou a predominância do gênero Haemonchus contortus.

Seis mesmas ovelhas de cada tratamento (duas por repetição) foram ordenhadas para cálculo da estimativa da quantidade $(\mathrm{kg})$ e posterior análise química do leite. As ordenhas foram realizadas semanalmente pelos mesmos ordenhadores a partir da quarta semana após o parto até a 11aㅡ $12^{\text {a }}$ semana, momento em que os cordeiros foram abatidos. Ovelhas com cordeiros gemelares foram ordenhadas enquanto os dois cordeiros estavam presentes, não sendo mais avaliadas após a saída de um dos cordeiros por atingir o peso de abate.

A técnica de ordenha empregada foi uma adaptação do método descrito por Lemos Neto e Cunha (1994). Realizou-se pré-ordenha das ovelhas até o total esvaziamento do úbere; neste momento, os cordeiros foram separados das mães por quatro horas, evitando assim as mamadas. Decorrido o período, 2 UI de ocitocina sintética foram administrados por via intramuscular profunda em cada ovelha, e após cinco minutos a ordenha manual foi cumprida de forma sistemática, esvaziando-se completamente cada teto de uma vez. O conteúdo obtido foi mensurado por meio de pesagem em balança eletrônica de precisão $(0,02 \mathrm{~g})$, sendo a produção em 24 horas estimada pela multiplicação por 6 (seis) do volume obtido nas quatro horas avaliadas. Uma alíquota de $3 \mathrm{~mL}$ do volume total homogeneizado de cada ovelha foi reservada em recipiente próprio com conservante bronopol, para posterior análise da composição pela técnica de análise por infravermelho. As amostras foram encaminhadas ao Laboratório do Programa de Análise de Rebanhos Leiteiros do Paraná, em Curitiba, para determinação de gordura, proteína, lactose e sólidos totais.

Para os resultados de produção e composição do leite foram utilizadas seis ovelhas por bloco, e, para os demais dados, nove ovelhas por bloco. Foi realizada análise de variância para modelos lineares (PROC GLM) no 
delineamento em blocos ao acaso, utilizando 0 tipo de parto como covariável, por meio do programa estatístico SAS (2001). Para comparação das médias de ovos de helminto por grama de fezes (OPG) entre os tratamentos, os dados foram transformados em $\log (x+1)$. Foi aplicado teste de Tukey a 5\% para comparação entre médias. Foi realizada análise de correlação simples de Pearson (PROC CORR) a $5 \%$ para as variáveis referentes à análise química do leite.

\section{RESULTADOS E DISCUSSÃO}

A média de peso inicial das ovelhas foi de 63,$06 ; 73,17$ e 70,38 kg de peso corporal nos sistemas com cordeiros mantidos em pastagem sem suplementação, com creep feeding e creep grazing, respectivamente, sem diferença $(P>0,05)$ entre os sistemas, como mostra a Tabela 2, na qual notase a perda de peso ocorrida durante 0 período.

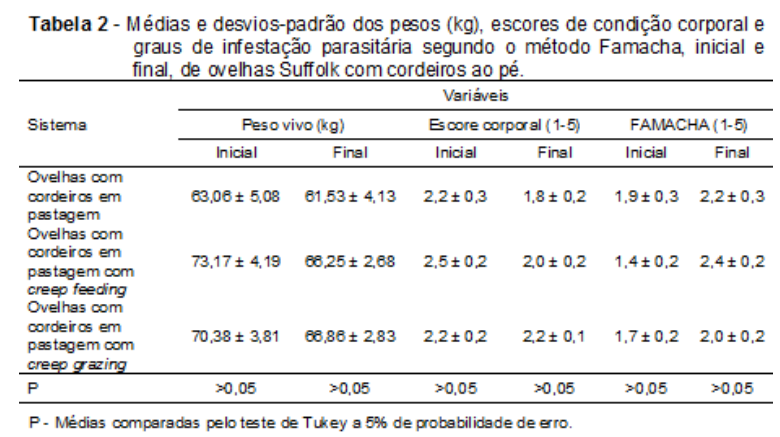

Este fato havia sido relatado por Podleskis et al. (2005) que demonstraram perda média de peso de $42 \mathrm{~g}$ por dia para ovelhas amamentando um único cordeiro e $107 \mathrm{~g}$ por dia para ovelhas amamentando gêmeos. Também Snowder e Glimp (1991) trabalhando com ovelhas Suffolk observaram perda de peso da ordem de 15 a $21 \%$ durante uma lactação de 96 dias, o que corrobora com os resultados observados no presente trabalho.
A perda de peso foi justificada pela incapacidade do pasto em suprir as elevadas exigências nutricionais nesta fase, de acordo com o NRC (2007). As ovelhas de maior peso sofreram maior perda de peso quando submetidas a uma pastagem de baixa qualidade, devido à maior exigência nutricional para mantença das mesmas (NRC, 2007).

Como mostra a Tabela 1, apesar da altura da pastagem estar dentro da altura recomendada, entre 14 e $16 \mathrm{~cm}$, para a categoria ovelhas em lactação, e da elevada massa de forragem total (entre 3800 a $4300 \mathrm{~kg}$ MS/ha), verificase que a quantidade de folhas disponíveis para as ovelhas (ao redor de 700 a $900 \mathrm{~kg} \mathrm{MSF} / \mathrm{ha}$ ) pode ter levado a este resultado de perda corporal, que é comumente citado para ruminantes na literatura (Meikle et al., 2004)

De outra forma, Ribeiro et al. (2009) trabalhando com ovelhas Suffolk com cordeiro ao pé em pasto de azevém, com e sem suplementação diária em creep feeding a $1 \%$ do PC do cordeiro, observaram ganho de peso das ovelhas de 82 e 79 g/dia, respectivamente. Os autores descreveram superior disponibilidade de folhas (mais de $1000 \mathrm{~kg} / \mathrm{ha}$ ), o que, ao contrário, pode ter sido fator limitante à seleção de forragem de qualidade pelas ovelhas, no presente estudo.

Houve efeito $(P=0,04) \quad$ da covariável tipo de parto sobre o escore corporal final das ovelhas, de tal forma que, a média do escore corporal para ovelhas de parto simples foi igual a 2,0 e para ovelhas de parto gemelar igual a 2,5, o que não era esperado. Sañudo e Montossi (2004) afirmaram que as ovelhas de parto gemelar sofrem mais influência da presença dos cordeiros em seu estado corporal. Provavelmente, isto aconteceu devido às ovelhas de parto simples terem saído da avaliação cerca de duas a três semanas antes das ovelhas de parto gemelar, utilizando a 

das ovelhas em pastagem

pastagem no período de florescimento do azevém. A formação de folhas na pastagem melhorou bastante no final do período experimental, entre Novembro e Dezembro, devido ao desenvolvimento vegetativo do Tifton-85. A massa de lâminas foliares da pastagem elevou-se de 601,25 $\pm 56,1 \mathrm{~kg} \mathrm{MS} / \mathrm{ha}$, em Setembro, até 1409,8 \pm 90,9 kg MS/ha em Dezembro, mostrando a importância da rebrota do pasto de Tifton - 85 em sistemas de terminação de cordeiros em pastos em sobressemeadura.

O grau Famacha apresentou pequena variação no período experimental, sem diferença significativa entre os tratamentos para Famacha inicial e final (Tabela 2). Os valores médios iniciais para as ovelhas $(1,9 ; 1,4$; 1,7 para sistemas sem suplementação, com creep feeding e com creep grazing, respectivamente) foram considerados bons, especialmente por estarem em lactação. Apesar da perda de peso e condição corporal, a variação do grau Famacha não foi acentuada, sendo que as ovelhas se mantiveram com grau Famacha próximo de 2,0 até Dezembro de 2007, o que determinaria a não aplicação de antiparasitários, de acordo com Molento et al. (2004).

Quanto à infestação parasitária determinada pelo número de ovos por grama de fezes (OPG), as ovelhas com cordeiros sem suplementação apresentaram a maior média de OPG entre os tratamentos no período experimental, com animais alcançando 4000 OPG (Figura 1).

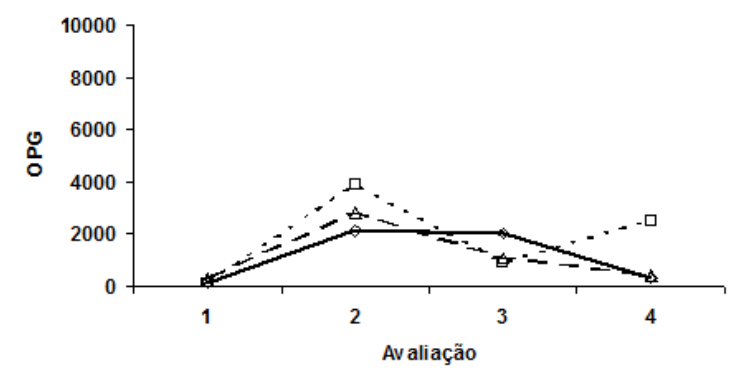

$\cdot-\square \cdot$ Sem Suplementação $\longrightarrow$ C reep Feeding $-\measuredangle-C$ reep Grazing

Figura 1 - Número de ovos por grama de fezes (OPG) para ovelhas em três sistemas de produção de cordeiros: cordeiros sem suplementação, creep feeding e creep grazing.
A análise estatística realizada com os dados transformados revelou diferença $(P<0,06)$ entre os sistemas, com maior infestação parasitária para o sistema sem suplementação $(1550,92$ OPG) quando comparado ao creep feeding (1063,88 OPG) e creep grazing $(1099,77$ OPG).

Este fato pode ser atribuído a pior condição nutricional das ovelhas do sistema não suplementado, em razão da limitada disponibilidade de folhas na pastagem durante a permanência dos animais na área. Esse aspecto é bastante prejudicial ao sistema de produção, especialmente nas condições de primavera chuvosa dessa região do Sul do Brasil, onde a contaminação larval na pastagem se eleva (Gazda et al., 2009).

A verminose pode acometer animais de qualquer sexo e idade, no entanto, o problema é mais severo em cordeiros (Colditz et al., 1996), e em fêmeas no periparto (Amarante et al., 1992), que compõe exatamente as categorias trabalhadas. Segundo Kahn et al. (2003) o aumento na contagem de OPG no periparto deve-se às mudanças hormonais associadas à supressão imunitária no período de lactação. Vieira et al. (2006) observaram ovelhas da raça Suffolk, Texel e lle de France puras e mestiças, em pastagem mista no Rio Grande do Sul e descreveram que os maiores valores de OPG ocorreram nos meses de Julho a Outubro (2300 OPG) coincidindo com o pico de lactação destas ovelhas.

A produção de leite das ovelhas variou $(P<0,05)$ em função do sistema de terminação (Figura 2). O tipo de parto não afetou a produção de leite, sendo esta variável apontada por Snowden e Glimp (1991) como a principal determinante do volume total de leite produzido pela ovelha. 


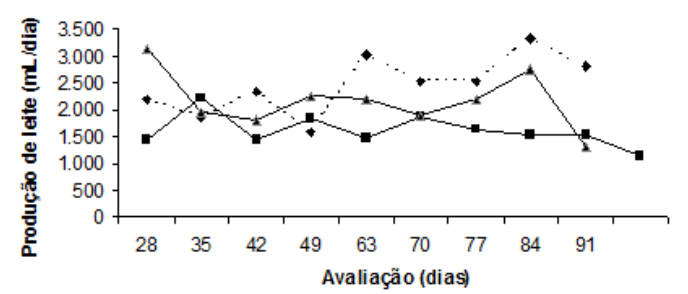

Figura 2 - Médias de produção de leite por ovelhas Suffolk em três sistemas de produção de cordeiros da quarta a décima primeira semana de lactação.

Ovelhas cujos cordeiros não recebiam suplementação produziram menos leite $(1,29 \pm 0,17 \mathrm{~kg} / \mathrm{dia})$ quando comparadas às ovelhas com cordeiros suplementados $(1,95 \pm 0,2 \mathrm{~kg} / \mathrm{dia}$, para creep feeding; $1,98 \pm 0,19 \mathrm{~kg} / \mathrm{dia}$, para creep grazing) que não diferiram entre si. Uma justificativa para as variações observadas no volume de leite produzido pelas ovelhas pode estar associada ao peso das ovelhas.

Outra justificativa para as diferenças no volume de produção de leite observado está associada ao desempenho dos cordeiros. De acordo com Emediato (2007), cordeiros maiores, que crescem mais rapidamente, mamam mais quando comparados aos cordeiros menores, e como consequência, a produção de leite das suas mães será maior. O ganho médio diário dos cordeiros nesse trabalho foi 0,$251 ; 0,324$ e $0,294 \mathrm{~g}$ por dia, respectivamente, para os tratamentos sem suplementação, com creep feeding e creep grazing. Nesse caso, os cordeiros de maior desenvolvimento estavam nos tratamentos com suplementação, o que possivelmente pode ter levado à maior produção de leite das mães.

O leite produzido pelas ovelhas não apresentou diferença significativa $(P>0,05)$ quanto a sua composição para as variáveis medidas. Este fato é justificado em grande parte pela condição homogênea da pastagem nos diferentes sistemas, uma vez que os fatores de ordem nutricional têm grande influência sobre a composição do leite.
Os valores em porcentagem (\%) de gordura, proteína, lactose e sólidos totais presentes no leite produzido pelas ovelhas estão representados na Tabela 3.

\begin{tabular}{|c|c|c|c|c|}
\hline Tratamentos & Gordura (\%) & Ladose (\%) & Proteina (96) & $\begin{array}{l}\text { Sólidos totais } \\
\text { (\%) }\end{array}$ \\
\hline $\begin{array}{l}\text { Ovelhas com cordeiros } \\
\text { semsuplemento }\end{array}$ & $6,01 \pm 0,98$ & $7.44 \pm 0.40$ & $4,12 \pm 0,48$ & $14,90 \pm 0,50$ \\
\hline $\begin{array}{l}\text { Ovelhas com cordeiros + } \\
\text { creep feeding }\end{array}$ & $5,01 \pm 1,18$ & $6,74 \pm 0.49$ & $3,20 \pm 0,58$ & $18,04 \pm 0,45$ \\
\hline $\begin{array}{l}\text { Ovelhas com cordeiros + } \\
\text { creep grazing }\end{array}$ & $5,22 \pm 1,13$ & $6,98 \pm 0,47$ & $3,67 \pm 0,57$ & $14,80 \pm 0,50$ \\
\hline$P$ & $P=0,6831$ & $P=0,3969$ & $P=0,3397$ & $P=0,2176$ \\
\hline
\end{tabular}

Os teores de gordura observados são semelhantes aos obtidos por Zimmermann et al. (2009) ordenhando ovelhas da raça Suffolk em pastagem de Brachiaria. Os autores encontraram teor médio de gordura no leite de $5,42 \%$, mostrando relativo aumento a partir da quinta semana de lactação, quando o volume de produção tendeu a reduzir, semelhante ao observado neste estudo.

Quanto à proteína do leite, esta apresenta correlação positiva com a porcentagem de gordura (OchoaCordero et al., 2002). Esse fato foi confirmado uma vez que as ovelhas que apresentaram maior teor de gordura tiveram-no para proteína $(r=0,81$; $\mathrm{P}<0,001)$.

Segundo Bencini e Pulina (1997) há correlação negativa entre produção e composição do leite. De acordo com os autores, a concentração de gordura, proteína e sólidos totais são maiores no inicio e final de lactação, e menores no pico, enquanto a concentração de lactose apresenta correlação direta com o volume de produção da lactação.

Entretanto, para as condições deste estudo, a diferença na produção de leite das ovelhas entre os sistemas, não teve magnitude suficiente a ponto de alterar a composição do leite. Este efeito pode ser mais facilmente evidenciado em raças especializadas em produção de leite, onde a nutrição 

das ovelhas em pastagem

tem maior impacto sobre a qualidade do mesmo (Chiofalo et al., 1993).

\section{CONCLUSÃO}

A suplementação de cordeiros em creep feeding e creep grazing não influenciou o desempenho ponderal e o estado corporal das ovelhas; porém, o melhor desenvolvimento dos cordeiros suplementados nesses sistemas promoveu a maior produção de leite das mães. O sistema de produção dos cordeiros influenciou o grau de infestação parasitária das matrizes.

\section{AGRADECIMENTOS}

Agradecemos ao CNPq - Conselho Nacional de Desenvolvimento Científico e Tecnológico pela bolsa Produtividade em Pesquisa, concedida a Alda Lúcia Gomes Monteiro, e pelo Auxílio à Pesquisa. Agradecemos a CAPES (Coordenação de Aperfeiçoamento de Pessoal de Nível Superior) às demais bolsas concedidas aos estudantes de Pós-Graduação.

\section{REFERÊNCIAS}

AMARANTE, A. F. T.; BARBOSA, M. A.; OLIVEIRA, M. A. G.et al. Efeito da administração de oxfendazol, ivermectina e levamisol sobre os exames coproparasitológicos de ovinos. Brazilian Journal of Animal Science, v.29, n.1, p.31-38, 1992.

BARTHRAM, G. T. Experimental techniques: The HFRO sward stick. Biennal Report 19841985. Hill Farming Research Organisation, Penicuik, p.29-30, 1986.

BENCINI, R.; PULINA, G. The quality of sheep milk: a Review. Wool Technology and Sheep Breeding, New Egland, v.45, p.182-220, 1997.

BENCINI, R.; PURVIS, I. W. The yield and composition of milk from Merino Sheep. Procedings of the Australian Society of Animal Production, v.18, p. 144-148, 1990.

BENCINI, R.; HARTMANN, P. E.; LIGHTFOOT, R. J. Comparative dairy potential of Awassi $x$
Merino and Merino ewes. Procedings of the Australian Association of Animal Breeding and Genetics, v. 10, p. 114-117, 1992.

CARVALHO, P. C. de F.; PONTES, L. S.; OLIVEIRA, E. O. et al. Sheep performance in Italian ryegrass swards at contrasting sward heights. In: INTERNATIONAL GRASSLAND CONGRESS, 19, Anais...Piracicaba, 2001 p.845-846.

CHIOFALO, V.; MICARI, P.; SAVOINI,G. et al. Impiego di differenti fonti energetiche per l'alimentazione dela pecorra: effetti sulle caratteristiche quanti-qualitative nel latte. Procedings of the 10th National ASPA (Assocoazone Scientifica Produzione Animale), Congress, p.339-344, 1993.

COLDITZ, I. G.; WATSON, D. I.; GRAY, G. D. et al. Some relationship between age, immune responsiveness and resistance to parasites in ruminants. International Journal for Parasitology, v.26, n. 8-9, p.869-877, 1996.

EMEDIATO, R. M. S. Efeito da gordura protegida sobre parâmetros produtivos de ovelhas da raça Bergamácia e na elaboração de queijos. 106p. Dissertação (Mestrado) Universidade Estadual Paulista, FMVZ, Botucatu, 2007.

FERNANDES, S.R.; MONTEIRO, A.L.G.; DITTRICH, R.L. et al. Early weaning and concentrate supplementation on the performance and metabolic profile of grazing lambs. Revista Brasileira de Zootecnia, v.41, n.5, p.1292-1300, 2012.

GAZDA, T. L.; PIAZZETTA, R.G.; DITTRICH, J.R. et al. Distribution of nematode larvae of sheep in tropical pasture plants. Small Ruminant Research, v. 82, n.2-3, p.94-98, 2009.

GIACCONE, P.; BIONDI, L.; BARRESSI, S. et al. Caratteristiche del sistema di allevamento degli ovini Comisani in Sicilia. Proceedings of the 10th Conference Italian Society of Patology and Farming of ovines and Caprines (SIPAOC), abs, 1992.

GORDON, H. M.; WHITLOCK, H. V. A new technique for counting nematode eggs in sheep faeces. Journal of the Council for Scientific and Industrial Research, New Delhi, v.12, p.50-52, 1939.

GREENWOOD, P. L.; HUNT, A.S. et al. Effects of birth weight and postnatal nutrition on 
neonatal sheep: II. Skeletal muscle growth and development. Journal of Animal Science, v.78, n.1, p.50-61, 2000.

KAHN, L.P.; KNOX, M. R.; WALKDEN-BROWN, S. W. et al. Regulation of the resistance to nematode parasites of single- and twin-bearing Merino ewes through nutrition and genetic selection. Veterinary Parasitology, v.114, n.1, p. 15-31, 2003.

LEMOS NETO, M.J. \& CUNHA, E.A. Comparação de métodos estimativos da produção de leite de ovelhas a pasto. Boletim de Indústria Animal, v.51, n.2, p.139-142, 1994.

MEIKLE, A.; KULCSAR, M.;CHILLIARD, Y. et al. Effects of parity and body condition at parturition on endocrine and reproductive parameters of the cow. Reproduction, v. 127, n.1, p. 727-737, 2004.

MOLENTO, M. B.; TASCA, C.; GALLO, A. et al. Método Famacha como parâmetro clínico individual de infecção por Haemonchus contortus em pequenos ruminantes. Ciência Rural, v.34, n.4, p.1139-1145, 2004.

MOTT, G. O.; LUCAS, H. L. The design, conduct and interpretation of grazing trials on cultivated and improved pastures. In: INTERNATIONAL GRASSLAND CONGREES, 6., 1952, State College. Proceedings...State College: Pensylvania State College Press, p. 1380-1385, 1952.

NRC - National Research Council. Nutrient requirements of small ruminants. Washington, D. C.: National Academy Press. 2007. 362 p.

OCHOA-CORDERO,M. A.;HERNÁNDEZ, G. T.; ALFARO, A. E. O. et al. Milk yield and composition of Rambouillet ewes under intensive management. Small Ruminant Research, v.43, n.3, p.269-274, 2002.

PODLESKIS, M. R.; RIBEIRO, E. L. A.; ROCHA, M. A. da et al. Produção de leite de ovelhas Hampshire Down e lle de France até os 84 dias de lactação. Semina: Ciências Agrárias, Londrina, v.26, n.1, p. 117-124, 2005.

POLI, C. H. E. C.; MONTEIRO, A. L. G.; BARROS, C. S. de et al. Produção de ovinos de corte em quatro sistemas de produção. Revista Brasileira de Zootecnia, v.37, n.4, p.666-673, 2008.
PULINA, G.; NUDDA, A.; RASSU, S. P. G. et al. Milk secretion during the day and the night in Sarda dairy ewes milked with different intervals. $45^{\text {th }}$ Annual Meeting European Association of Animal Production, Edinburgh (UK), p. 195 196, 1994.

RIBEIRO, T.M.D.; MONTEIRO, A.L.G.; POLI, C.H.E.C. et al. Características da pastagem de azevém e produtividade de cordeiros em pastejo. Revista Brasileira de Zootecnia, v.38, n.3, p.580-587, 2009.

RUSSEL, A. J. F.; DONEY, J. M.; GUNN, R. G. Subjective assessment of body fat in live sheep. Journal Agricultural Science, Cambridge, v.72, n.3, p.451-454, 1969.

SAÑUDO, C.; MONTOSSI, F. Evaluación y promoción de la calidad de la carne y otros productos agroalimentarios uruguayos en base a los estándares de calidad de la Unión Europea y en función de distintos sistemas productivos del Uruguay - Informe Final del Proyeto Componente Carnes - Septiembre, 2004, 56p.

SAS Institute. System for Information. Versão 8.2, Carry, 2001. 8 CD-ROM.

SNOWDER, G. D.; GLIMP, H. A. Influence of breed, number of suckling lambs, and stage of lactation on ewe Milk production and lamb growth under range conditions. Journal of animal Science, v.69, n.3, p.923-930, 1991.

SERRA, A.; MACCIOTTA, N. P. P.; NUDDA, A. et al. L'influenza dela técnica di alimentazione per gruppi sulla qualita del latte negli ovini.

Procedings of the Italian Society of Veterinary Science, v.47,p .207-210, 1993.

VIEIRA, M. I.; OLIVEIRA, I. S.; ROCHA, H. C. et al. Controle seletivo do Haemonchus contortus em ovinos através do método Famacha. IN: XXXIV Jornadas Uruguayas de Buiatria, Anais... Paysandu UY. 2006, p.166-167.

ZIMMERMANN, N. P; MONREAL, A. C. D; OLIVEIRA, J. V. et al. Controle leiteiro e análise centesimal do leite de ovelhas Suffolk. Arq. Ciênc. Vet. Zool. Unipar, v.12, n.1, p.37- 45, 2009. 